\title{
Semi-active Pneumatic Devices for Control of MDOF Structures
}

\author{
Y. Ribakov*
}

Department of Civil Engineering, Ariel University Center of Samaria, Israel

\begin{abstract}
A preliminary study of a semi-active control system with pneumatic devices, aimed to improve the response of multi-degree of freedom structures to earthquakes, is presented. The devices are attached at various levels of a structural frame. The aim of the design is to find a combination of forces that are produced by the devices, in order to obtain optimal structural response. The mechanical response of the devices is regulated by air pressure, which is varied in each device at every time step in such a way that the forces in the devices will be close as possible to those, obtained by the optimization procedure. Linear auto-regressive model with exogenous input is used to predict the displacements and the velocities of the frame in order to overcome the time delay in the control system. The control forces are calculated at every time step, using the instantaneous optimal control algorithm, according to the values of the displacements and of the velocities that are predicted for the next time step, for each story of the structure. Numerical analysis of a seven story structure with pneumatic devices was carried out. It shows a significant improvement of the structural response when the proposed control system is applied, compared to that of an uncontrolled structure and of a structure with semi-active controlled electrorheological dampers.
\end{abstract}

\section{INTRODUCTION}

Passive energy dissipating systems, such as viscous dampers, tuned mass dampers and base isolation systems have been installed in new and existing buildings all over the world. It has resulted in improved structural response to earthquakes. However, active systems have wider ranges of operation, as shown in state-of-the-art publications $[1,2]$.

More than ten years ago Soong [3], Agrawal et al. [4] and other researchers have reported that devices, using external energy and algorithmic logic, can produce more optimal reductions than passive systems. Gavin et al. [5] and Makris et al. [6] have proposed to use electrorheological (ER) fluid dampers as a part of semi-active systems for seismic applications. They have also developed theoretical models, allowing effective prediction of hysteretic behavior for such dampers.

Ribakov et al. [7] have shown that semi - active control systems, including ER dampers, designed according to the instantaneous optimal control theory [8], significantly improve the behavior of buildings during earthquake motions. It was also shown that the time delay problem in the control system can be successfully solved, using a predictive control algorithm [9]. Following this technique, in this study the control forces are calculated, using instantaneous control theory, according to the predicted values of the displacements and velocities for the next time step (rather than at their occurring values).

Magnetorheological (MR) dampers were proposed as alternative devices to the ER ones [10, 11]. Many studies have been recently carried out on this type of dampers. Xu et al. [12] proposed an on-line real-time control method for

*Address correspondence to this author at the Department of Civil Engineering, Ariel University Center of Samaria, Ariel, Israel; Tel: +972-546431 385: Fax: +972 390663 51: E-mail: ribakov@ariel.ac.il semi-active control of structures with MR dampers and used the Levenberg-Marquardt algorithm to train the on-line control neural network. The method considers time-delay in the semi-active controlled system. A comparison between controlled structure and uncontrolled one showed that the online real-time control method is effective for use in structures with MR dampers. and that the Levenberg-Marquardt algorithm has a very fast convergence rate. Yang et al. [13] presented a dynamic model of an MR damper and power supplying system. Force response analysis of the MR damper was performed, and a model for estimation the damper's behavior under dynamic loading has been proposed.

A comprehensive review of designed techniques, providing engineers with the knowledge, needed for successful implementation of active, hybrid and semi-active control systems in structures for safeguarding them against earthquakes, was done by Chu et al. [14]. Recently Preumont and Seto [15] presented the state-of-the-art in theory, design and application of active vibration control. They introduced active vibration control through the use of smart materials and structures, semi-active control devices and a variety of feedback options and discussed many interesting and useful topics, including main methods and devices used in high-rise buildings.

In this study an alternative Semi-Active Pneumatic Device (SAPD) is proposed to be implemented in structures in order to improve their response to earthquakes. The current paper includes description of the proposed device and the use of such devices, placed between Chevron braces and the rigid floor diaphragms (Fig. 1a).

The forces that can be developed in the pneumatic dampers, are of practical order of magnitude, making them suitable for structural engineering applications. These forces are obtained by varying the pressure in the damper at each time step according to the values that are calculated for displacements and velocities, which are expected to occur after a 
certain time (taking into account the time delay in the system).

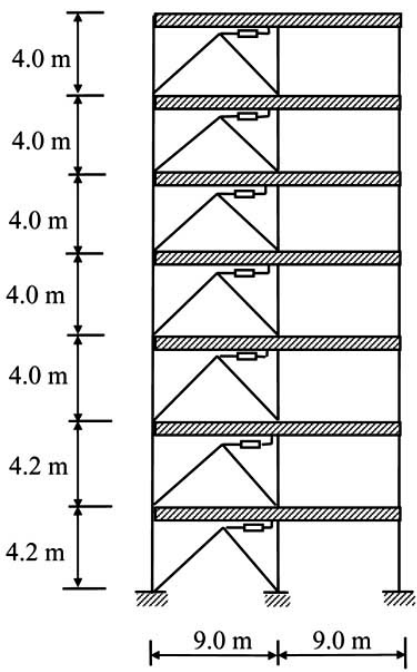

(a)

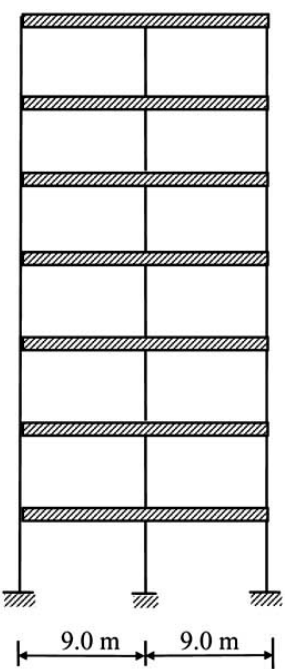

(b)
Fig. (1). A seven-story structure (a) damped, (b) uncontrolled.

\section{CONSTRUCTION AND CHARACTERISTICS OF A PNEUMATIC DEVICE}

A general scheme of the device is shown in Fig. (2). Following the figure, the device consists of a cylinder (1), a piston (2) with a rod (3), an air pressure reservoir (4), two control valves $(5 \mathrm{a}, 5 \mathrm{~b})$, and two valves $(6 \mathrm{a}, 6 \mathrm{~b})$, connecting the cylinder to the atmosphere. The devices are installed at each floor of the structure by connecting the cylinder (' 1 ' in Fig. 2) to the Chevron brace, and the rod (' 3 ' in Fig. 2) to the structure's upper floor diaphragm.

It should be mentioned that the forces that may be applied to a structure by ER or MR dampers are always opposite to drift velocities. The SAPDs allow application of control forces in both directions, in other words they may have an effect of a certain negative stiffness and due to this feature it is expected to obtain further improvement in the structural response.

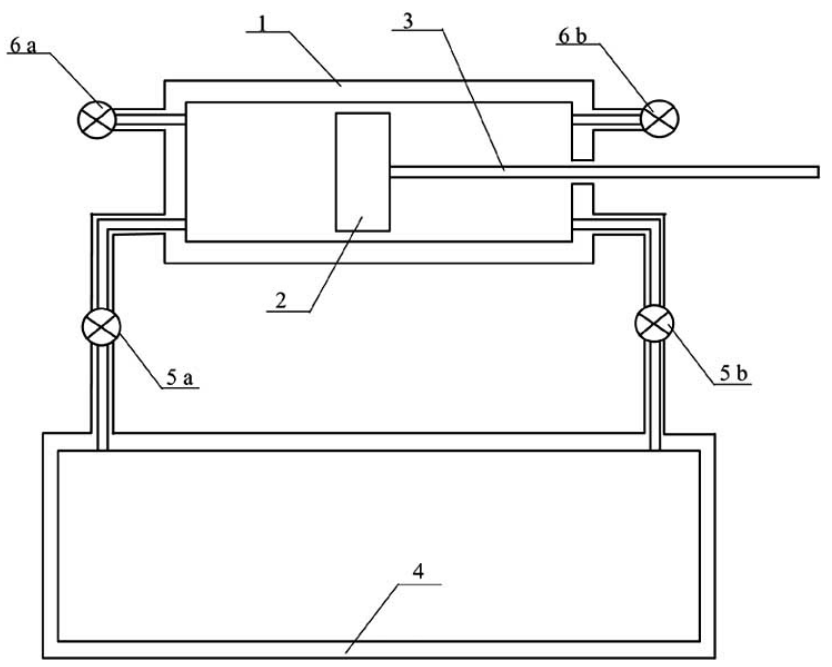

Fig. (2). A general scheme of a SAPD.
When the required optimal control force should act in a direction opposite to drift velocity, it can be contributed by the stiffness of the device $k$ that can be obtained similar to that of a pneumatic spring [16]:

$k=\frac{n P_{i} S^{2}}{V_{i}}\left(\frac{1}{1-\left(S / V_{i}\right) d}\right)^{n+1}$

$P_{i}$ is the air pressure at the piston's reference displacement, $d$

$\mathrm{S}$ is the piston's area,

$V_{i}$ is the corresponding volume of the contained air, and $n$ is the heats ratio ( $n=1.4$ for air).

If the change in volume is small relative to the initial volume, then Eq. (1) can be reduced as follows:

$k=\frac{n P_{i} S^{2}}{V_{i}}$

Using the control valves ( $5 \mathrm{a}$ and $5 \mathrm{~b}$ ), a necessary pressure is transferred to one of the cylinder's chambers, and the stiffness of the device is adjusted in order to obtain the force, equal to its optimal value.

But sometimes, according to the optimal control law, the control force should act in the direction of drift velocity. SAPDs offer such possibility. In this case the force, applied by the device to the structure, is proportional to the piston's area $\mathrm{S}$ and to the pressure $\mathrm{P}$. Although the pressure and the area are limited, but this feature allows a certain negative stiffness that is expected to lead further improvement in structural seismic response.

The pressure in SAPD cannot be changed instantaneously, because a certain time is required to obtain the desired pressure when the valve is opened. That is why the stiffness that yields the optimal control forces at each floor is evaluated according to the displacements, obtained by the prediction technique [9]. The overall behavior of the SAPD is similar to a nonlinear spring with variable stiffness (positive or negative) having different, controllable loading and unloading curves, which enables energy dissipation.

The proposed type of SAPD, used in the current work, has an additional advantage, that forces in the device do not change instantaneously when the velocity changes sign (like in friction dampers), and the power, required to activate the SAPD during the earthquake, is small enough, making it attractive for practical applications.

\section{CONTROL ALGORITHM}

A response of a structure, provided with supplemental dampers, is described by the following dynamic equation of equilibrium [3]:

$M \ddot{x}(t)+C \dot{x}(t)+K x(t)=L f_{e}(t)+D u(t)$

where $M, C$ and $K$ are the mass, damping, and stiffness matrices, respectively,

$x(t), \dot{x}(t), \ddot{x}(t)$ are the displacement, velocity and acceleration vectors, respectively,

$u(t)$ is the vector of forces in the supplemental devices,

$f_{e}(t)$ is the external excitation, 
$D$ and $L$ are the control and excitation forces matrices, respectively.

The system of differential equations (3) can be simplified by transformation into the space state form:

$\dot{z}(t)=A z(t)+B u(t)+H f_{e}(t)$

where $z(t)=[x(t), \dot{x}(t)]^{T}$ is the 2 n state space vector of the displacements and velocities of the structure, and $A$ is the system matrix, given by:

$A_{2 n \times 2 n}=\left[\begin{array}{cc}0 & 1 \\ -M^{-1} K & -M^{-1} C\end{array}\right]$

and $B$ and $H$ are matrices, specifying, respectively, the locations of controllers and external excitations in state space:

$B_{2 n \times m}=\left[\begin{array}{c}0 \\ M^{-1} D\end{array}\right]$ and $H_{2 n \times r}=\left[\begin{array}{c}0 \\ M^{-1} L\end{array}\right]$

Considering a closed-loop instantaneous control system (Soong, 1990) the control forces vector at each time step, $t$, is obtained as follows:

$u(t)=-\frac{\Delta t}{2} R^{-1} B^{T} Q z(t)$

where $\Delta t$ is the time increment, and $R$ and $Q$ are weighting matrices, whose magnitudes are assigned according to the relative importance, attached to the state variables and to the control forces [3]:

$Q=I_{2 n \times 2 n}$

$R=10^{-m} I$

$I$ is a $2 \mathrm{n} \times 2 \mathrm{n}$ unit diagonal matrix, and $m$ is a parameter which is used to tune the system within a practical range.

The response state vector is

$z(t)=\left[I+\frac{\Delta t^{2}}{4} B R^{-1} B^{T} Q\right]^{-1}\left[T d(t-\Delta t)+\frac{\Delta t}{2} H f_{e}(t)\right]$

and

$d(t-\Delta t)=\exp (\Lambda \Delta t) T^{-1}\{z(t-\Delta t)+$

$\left.\frac{\Delta t}{2}\left[B u_{c}(t-\Delta t)+H f_{e}(t-\Delta t)\right]\right\}$

where $T$ is a $2 n \times 2 n$ modal matrix, whose columns are eigenvectors of $\mathrm{A}$, and $\Lambda$ is a diagonal matrix whose diagonal elements are the complex eigenvalues of the matrix $A$ :

$\Lambda=T^{-1} A T$

Instantaneous application of the optimal forces yields significant improvement in the structure's behavior [7]. However, in practice there is a delay from the time of the forces calculation until they are applied. In other words, the response of a structure with the proposed SAPD is measured and the signals are transferred to the computer, calculating the control forces that should be applied instantaneously. But until the calculated control is applied to yield the desired damping forces, the behavior of the structure changes and indeed the damping is already not optimal. Due to this delay, the behavior of the structure may be even worse, compared to the uncontrolled one. To avoid this phenomenon predictive control is used. According to the predictive control approach [9], the structure's response (displacements and velocities) is evaluated at the time, when the control forces are expected to be applied. Thus, the control forces are calculated according to their actual application time.

Assuming that the input signal, $f_{e}(t)$, and the output signal, $z(t)$, are related by a linear system, the relationship can be written as follows [17]:

$z(t)=G(q) f_{e}(t)+v(t)$

where $G(q)$ is the "transfer function" of the system, and $v(t)$ is an additional disturbance.

$G(q)=\sum_{k=1}^{\infty} g(k) q^{-k}$

$G(q) f_{e}(t)=\sum_{k=1}^{\infty} g(k) f_{e}(t-k \Delta t)$

$q$ and $q^{-1}$ denote, respectively, shift and delay operators, where

$q^{-1} f_{e}(t)=f_{e}(t-\Delta t)$

The numbers $g(k)$ in Eq. (15) are called the impulse response of the system at time step $k$.

A commonly used linear black box parametric model is the ARX model with exogenous input [18]:

$\tilde{A}(q) z(t)=\tilde{B}(q) f_{e}\left(t-n_{k} \Delta t\right)+e(t)$

where $\tilde{A}$ includes the auto-regressive parameters, $\tilde{B}$ is the exogenous part, and the time delay from the input to output is equal to $n_{k} \Delta t$.

$\tilde{A}$ and $\tilde{B}$ are polynomials in the delay operator $q^{-1}$ :

$\tilde{A}(q)=1+a_{1} q^{-1}+\ldots+a_{n a} \quad q^{-n_{a}}$

$\tilde{B}(q)=b_{1}+b_{2} q^{-1}+\ldots+b_{n_{b}} q^{-n_{b}+1}$

Here $n_{a}$ and $n_{b}$ are the orders of the polynomials.

Application of the shift operator, $q$, (Eq. 16), with $z(t)$ (Eq. 13) with the polynomials $\tilde{A}(q)$ and $\tilde{B}(q)$ (Equations 18 and 19) leads to:

$\tilde{A}(q) z(t)=z(t)+a_{1} z(t-\Delta t)+\ldots+$

$a_{n_{a}} z\left(t-n_{a} \Delta t\right)$

$\tilde{B}(q) f_{e}\left(t-n_{k} \Delta t\right)=$

$b_{1} f_{e}\left(t-n_{k} \Delta t\right)+b_{2} f_{e}\left[t-\left(n_{k}+1\right) \Delta t\right]+$

$b_{n_{b}} f_{e}\left[t-\left(n_{k}+n_{b}-1\right) \Delta t\right]$

Eq. (17) can be now rewritten in an explicit form as follows:

$z(t)+a_{1} z(t-\Delta t)+\ldots+a_{n_{a}} z\left(t-n_{a} \Delta t\right)=$

$b_{1} f_{e}\left(t-n_{k} \Delta t\right)+b_{2} f_{e}\left[t-\left(n_{k}+1\right) \Delta t\right]+\ldots+$

$b_{n_{b}} f_{e}\left[t-\left(n_{k}+n_{b}-1\right) \Delta t\right]+e(t)$ 
By analyzing the building's response at discrete time increments during the earthquake, optimal forces in the dampers at every structural level are calculated according to the predicted values of the displacements and velocities. If a structure is provided by ER or MR dampers and the required optimal control force should be applied in the direction of drift velocity, zero control force is applied. If the optimal force is larger then the maximum value that can be practically achieved in an ER or MR damper, then maximum electric or magnetic field is applied.

For the case when SAPD are used, the air pressure in each device is varied in such a way that it produces at time $t$ the forces that were obtained by the optimization procedure at time $\left(t-n_{k} \Delta t\right)$ (according to the predicted displacements at time $t$ ). If the optimal force is larger then the maximum force that can be produced by the SAPD for the predicted displacement value, then maximum pressure is applied.

An additional issue that should be discussed is the number and nature of physical quantities to be measured. As it follows from the instantaneous control algorithm, described above, the state space vector $\mathrm{z}(\mathrm{t})$ includes displacements and velocities at all floors. In the frame of this study the values of displacements and velocities are calculated, hence there is no problem to use a full feedback for the control algorithm. But in real applications measuring of all structural displacements and velocities is not always possible. If the feedback cannot be measured at all, control algorithms with limited feedback are applied [19].

\section{NUMERICAL EXAMPLE}

In order to investigate the effectiveness of the proposed devices, simulations of a seven story framed structure with infinitely stiff beams were carried out (Fig. 1). The response was computed for the following four seismic excitations:

El-Centro (1940),

Northridge (1994),

Loma-Prieta (1989), and

Kobe (1995).

The second and the last were chosen as motions with high peak ground acceleration, yielding nonlinear deformations and big accelerations in the structure. All simulations were performed using routines written in MATLAB.

The structure was characterized by the following matrices:

$M=87.5 I_{7 \times 7}[$ ton]

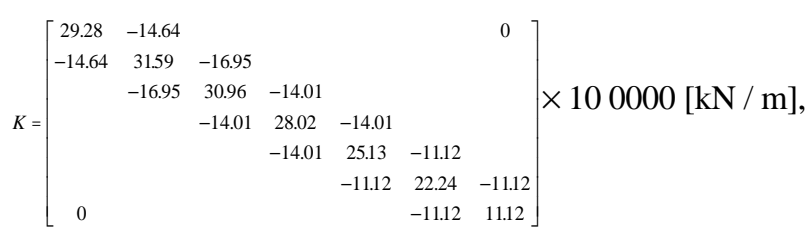

where $M$ is the mass matrix of the structure,

$I$ is a unit diagonal matrix, and

$K$ is the structure's stiffness matrix.

An initial damping ratio of $1 \%$ was assumed for all vibration modes of the uncontrolled structure. At each time step of an occurring earthquake the MATLAB System Identification Toolbox was used to calculate the ARX model in order to predict the structure's displacements and velocities at the next time step. These predicted values were used to obtain the optimal control forces in the dampers according to the control theory.

Numerical analyses of the structure were performed for the following three cases:

- an uncontrolled structure,

- a structure with ER dampers, and

- a structure with the proposed SAPD.

The control forces in the dampers, used in the second and third cases, were obtained according to the control algorithm that was described in the previous section. The maximum control force in the ER and SAPD as assumed to be less than $250 \mathrm{kN}$.

As it was mentioned above, the forces in the ER dampers are always opposite to drift velocities. The SAPDs allow application of forces in both directions, in other words they may have an effect of a certain negative stiffness and due to this feature it is expected to obtain further improvement in the structural response.

Peak displacements and base shear forces of the uncontrolled structure, of the ER damped structure, and of the structure with SAPD are presented in Tables 1, 2 and $\mathbf{3 .}$

Table I. Peak Displacements [cm] and Base Shear Forces $[\mathrm{kN}]$ in the Uncontrolled Structure

\begin{tabular}{|c|c|c|c|c|}
\hline Story & Elcentro & Northridge & Loma-Prieta & Kobe \\
\hline \hline 7 & 12.85 & 24.84 & 16.35 & 63.84 \\
\hline 6 & 12.07 & 23.55 & 15.59 & 60.19 \\
\hline 5 & 10.56 & 21.01 & 14.04 & 53.13 \\
\hline 4 & 8.83 & 18.01 & 12.11 & 44.97 \\
\hline 3 & 6.72 & 14.16 & 9.56 & 34.82 \\
\hline 2 & 4.76 & 10.37 & 7.02 & 25.14 \\
\hline 1 & 2.45 & 5.39 & 3.66 & 12.91 \\
\hline BS & 1753 & 5451 & 2796 & 6609 \\
\hline
\end{tabular}

Reductions of up to $60 \%$ and $70 \%$ were obtained in the peak displacements of the structure with ER dampers and with SAPD, respectively (see Tables 1, 2, and 3). Additionally, according to the analytical results that were obtained in the frame of this study, using the SAPD yields significant reduction in structure's accelerations, compared to that of the uncontrolled and ER damped structure. As it follows from the tables, there was no significant change of the peak base shear forces of the structure with the SAPD, compared to those of the uncontrolled structure, whereas for the ER damped structure the base shear forces increased under Northridge and Loma - Prieta earthquakes. 
Table 2. Peak Displacements [cm] and Base Shear Forces $[\mathrm{kN}]$ in the Structure with ER Dampers

\begin{tabular}{|c|c|c|c|c|}
\hline Story & Elcentro & Northridge & Loma-Prieta & Kobe \\
\hline \hline 7 & 5.31 & 18.76 & 9.72 & 34.81 \\
\hline 6 & 5.14 & 18.01 & 9.32 & 32.28 \\
\hline 5 & 4.84 & 16.20 & 8.45 & 28.76 \\
\hline 4 & 3.76 & 14.13 & 7.31 & 24.51 \\
\hline 3 & 2.92 & 11.31 & 5.80 & 19.12 \\
\hline 2 & 2.20 & 8.37 & 4.28 & 13.89 \\
\hline 1 & 1.10 & 4.50 & 2.25 & 7.19 \\
\hline BS & 1802 & 5718 & 3210 & 5850 \\
\hline
\end{tabular}

Table 3. Peak Displacements $[\mathrm{cm}]$ and Base Shear Forces $[k N]$ in the Structure with SAPD

\begin{tabular}{|c|c|c|c|c|}
\hline Story & Elcentro & Northridge & Loma-Prieta & Kobe \\
\hline \hline 7 & 4.05 & 16.20 & 7.31 & 20.62 \\
\hline 6 & 3.82 & 15.31 & 6.96 & 19.53 \\
\hline 5 & 3.38 & 13.76 & 6.27 & 17.94 \\
\hline 4 & 2.83 & 11.90 & 5.45 & 14.96 \\
\hline 3 & 2.16 & 9.53 & 4.37 & 11.74 \\
\hline 2 & 1.55 & 7.08 & 3.26 & 8.57 \\
\hline 1 & 0.74 & 3.90 & 1.75 & 4.43 \\
\hline BS & 1805 & 5460 & 2770 & 6520 \\
\hline
\end{tabular}

\section{CONCLUSIONS}

New semi-active pneumatic dampers were proposed to improve structural response to earthquakes. A procedure was developed for a semi-active control system with these pneumatic devices. Predictive active control theory was applied to find the optimal control forces. The optimal control forces in the pneumatic devices were calculated during an earthquake and applied simultaneously with their corresponding displacements and velocities at the next time step (without delay).

A numerical simulation of a seven story framed building was performed for the following three cases: uncontrolled structure, a structure with ER dampers, and a structure with the proposed semi-active pneumatic devices. The simulation shows effective reductions in the peak displacements and peak accelerations without a significant difference of the base shear forces in a structure with pneumatic devices com- pared to that of the uncontrolled structure and to the ER damped one. The simulation also shows that for certain earthquake time histories, especially for near future ground motions, significant damage in a structure may be prevented by using the proposed semi-active control system. Experimental validation of the numerical results, obtained in this preliminary study, is required. It can be done by testing a small-scale structural model.

\section{REFERENCES}

[1] G. Housner, "Structural control: past, present and future", ASCE Journal of Engineering Mechanics, vol. 123(9), pp. 897-971, 1997.

[2] L. S. Fur, H. T. Y. Yang and S. Ankireddi, "Vibration control of tall buildings under seismic and wind loads", ASCE Journal of Structural Engineering, vol. 122(8), pp. 948-57, 1996.

[3] T. T. Soong, Active Structural Control: Theory and Practice. Longman Scientific \& Technical: UK, 1990.

[4] A. K. Agrawal and Yang J. N, "Optimal polynomial control of seismically excited linear structures", ASCE Journal of Engineering Mechanics, vol. 122(8), pp. 753-761, 1996.

[5] H. P. Gavin, R. D. Hanson and N. H. Mc-Clamroch, Control of structures using electrorheological dampers, Eleventh World Conference on Earthquake Engineering, Pergamon, Elsevier Science Ltd., Oxford, England, Paper No. 272, 1996.

[6] N. Makris, S. A. Burton, D. Hill and Jordan M, "Analysis and design of an ER damper for seismic protection of structures", ASCE Journal of Engineering Mechanics, vol. 122, pp. 1003-1011, 1996.

[7] Y. Ribakov and J. Gluck, "Active control of MDOF structures with supplemental electrorheological fluid dampers", Earthquake Engineering and Structural Dynamics, vol. 28, pp. 143-156, 1999.

[8] J. N. Yang, A. Akbarpour and P. Ghemmaghami, "New optimal control algorithms for structural control", ASCE Journal of Engineering Mechanics, vol. 113(9), pp. 1369-1386, 1987.

[9] J. Gluck, Y. Ribakov and A. N. Dancygier, "Predictive active control of MDOF structures", Earthquake Engineering and Structural Dynamics, vol. 29, pp.109-125, 2000.

[10] J. D. Carlson and M. J. Chrzan, "Magnetorheological fluid dampers", U.S. Patent 5,277,281, 1994.

[11] J. D. Carlson, M. J. Chrzan and F. O. James, "Magnetorheological fluid devices", U.S. Patent 5,398,917, 1995.

[12] Z. D. Xu, Y. P. Shen and Y. Q. Guo, "Semi-active control of structures incorporated with magnetorheological dampers using neural networks", Smart Materials and Structures, vol. 12, pp. 80-87, 2003.

[13] G. Yang, B. F. J. Spencer, H. J. Jung and J. D. Carlson, "Dynamic modeling of large-scale magnetorheological damper systems for civil engineering applications", ASCE Journal of Engineering Mechanics, vol. 130(9), pp. 1107-1114, 2004.

[14] S. Y. Chu, T. T. Soong and A. M. Wiley, Reinhorn, Active, Hybrid, and Semi-active Structural Control A Design and Implementation Handbook, Wiley: USA, 2005.

[15] A. Preumont and K. Seto, Active Control of Structures, Wiley, 2008.

[16] C. M. Harris and C. E. Crede, Shock and Vibration Handbook, McGraw-Hill Inc., NY, 1976.

[17] L. Ljung, System Identification Toolbox User's Guide, The Math Works Inc. 1997.

[18] L. Ljung, System Identification: Theory for User, Prentice-Hall: Englewood Cliffs, New Jersey, USA, 1987.

[19] W. Levine, T. Johnson and M. Athans, "Optimal limited state variable feedback controllers for linear systems", IEEE Transactions on Automatic Control, vol. 16(6), pp. 785-793, 2003. 